\title{
lieuxdits \#6
}

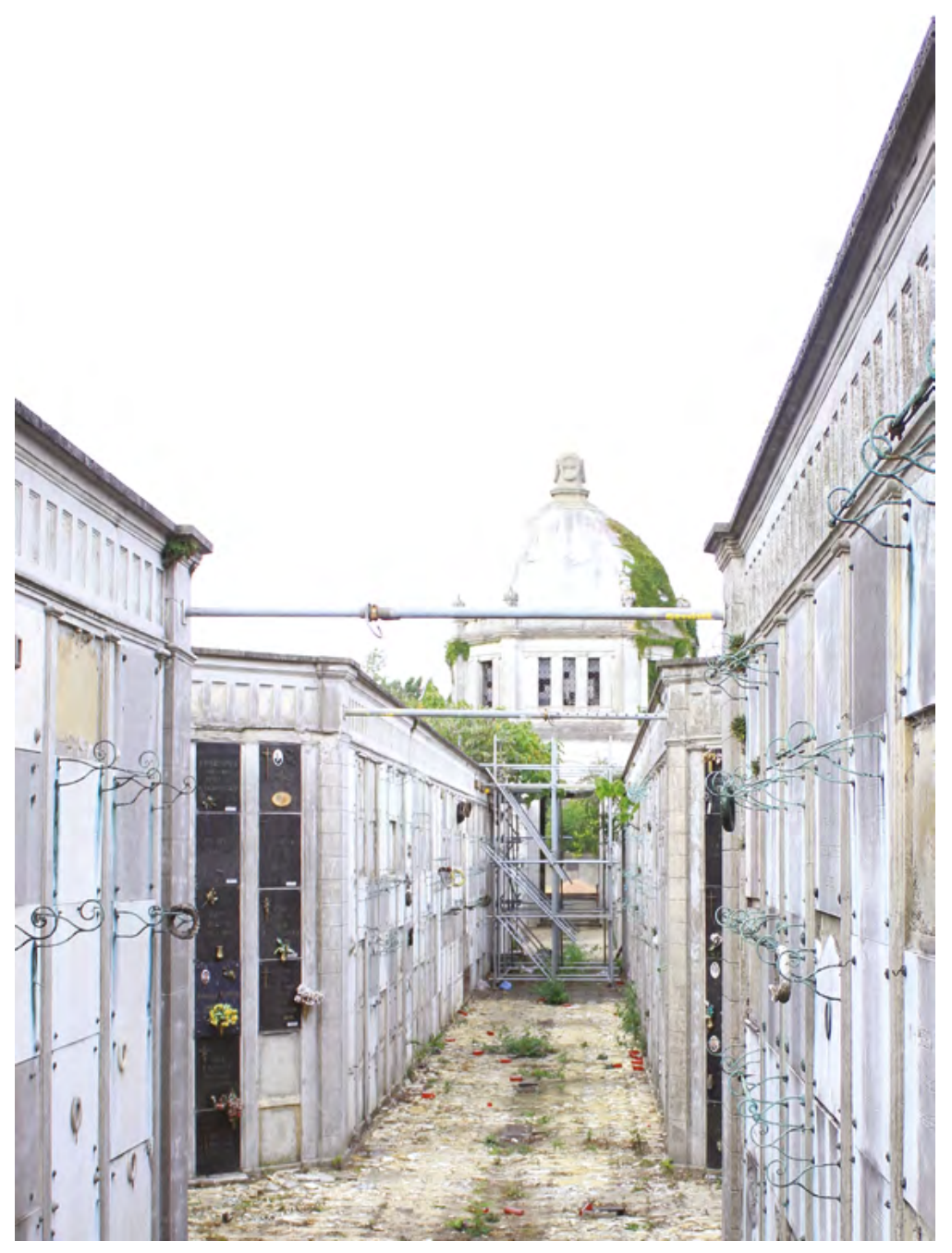

Référence bibliographique :

Geert De Groot, "Le formalisme face à l'iconographie", lieuxdits\#6, novembre 2013, pp.8-13.

\section{La revue lieuxdits}

Faculté d'architecture, d'ingénierie architecturale, d'urbanisme (LOCI)

Univesrité catholique de Louvain (UCL).

Éditeur responsable : Jean-Paul Verleyen, place des Sciences, 1 - 1348 Louvain-la-Neuve

Comité de rédaction : Martin Buysse, Damien Claeys, Gauthier Coton,

Jean-Philippe De Visscher, Guillaume Vanneste, Jean-Paul Verleyen

Conception graphique : Nicolas Lorent

Impression : école d'imprimerie Saint-Luc Tournai

\section{(c) (1) () $\Theta$}




\section{Le formalisme face à l'iconographie}

Trois projets de Konstantin Stepanov Melnikov

Geert De Groote

Les années d'étude de Melnikov coïncidèrent avec le tourbillon de changements auquel la Russie fut confrontée au début du XXe siècle. Melnikov étudia la peinture et l'architecture à l'académie de Moscou, qui deviendra plus tard les célèbres ateliers Vkhoutemas. Ses premiers mentors artistiques lui firent découvrir le langage formel classique, conforme au goût conservateur de l'establishment. Symétrie et monumentalité furent les maîtres mots de toute son œuvre. II termina ses études d'architecture en 1917, au début de la Révolution russe. Si le jeune Konstantin se sentait attiré vers la peinture et l'architecture traditionnelle, son âme rebelle était en quête de nouvelles influences. Rapidement, il succomba à l'emprise de la contre-culture de la capitale.

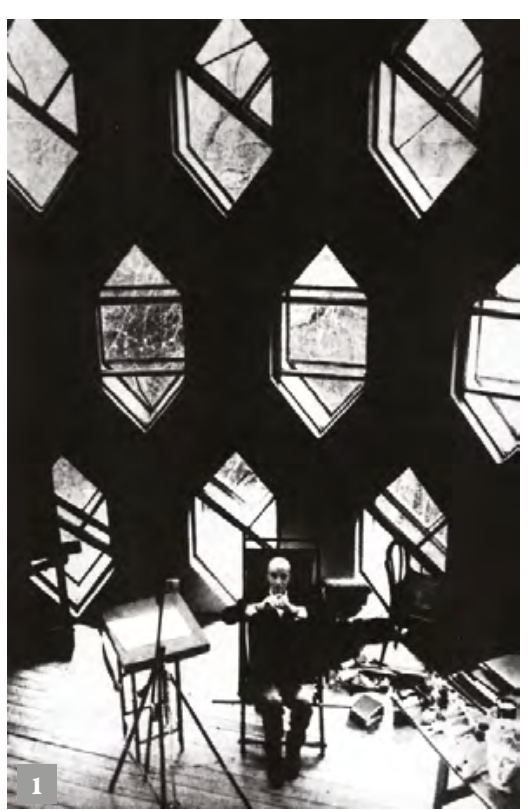

Au même moment, dans toute l'Europe, des artistes se révoltaient contre l'ordre établi. Ils croyaient fermement en la réalisation d'une société nouvelle, en rupture totale avec le passé. C'est ainsi que vit le jour une architecture dénuée d'ornements, au service de la communauté, qui prêchait pour la géométrie et la fonctionnalité. La subjectivité laissa place à l'objectivité. La pénurie de logements fut prise au sérieux et tous les sacrifices furent bons pour la contrer. En raison des nécessités économiques, mêmes les plus grands détracteurs de l'architecture cubiste naissante trouvèrent justifiée cette extrême sobriété formelle. ${ }^{1} \mathrm{En}$ d'autres termes, elle devait permettre de combler le fossé entre les besoins et les possibilités.

Pour les architectes russes, le problème était plus compliqué. Le pays resta enlisé dans la guerre civile jusqu'à la fondation de l'Union soviétique en 1922, ce qu entraîna une destruction économique. Toutefois, les bouleversements politiques et le manque aigu de moyens financiers alimentèrent le débat théorique et l'expérimentation. Influencée par une vision révolutionnaire de la société, l'architecture traditionnelle dut faire place aux exercices de style constructiviste. Tatline conçut un extravagant monument à la Troisième Internationale, une structure d'acier plus haute que la Tour Eiffel et dotée de blocs de bureaux en rotation. El Lissitzky imagina l'immeuble Wolkenbügel, dont la superficie utilisable dépassait à peine la surface de circulation. Malevitch joua avec des blocs et inventa ainsi l'architecture aveugle. La mégalomanie de ces projets utopiques et irréalisables contrastait douloureusement avec les besoins de la population. La nation passait par une phase de transition, l'architecture était en ménopause. 


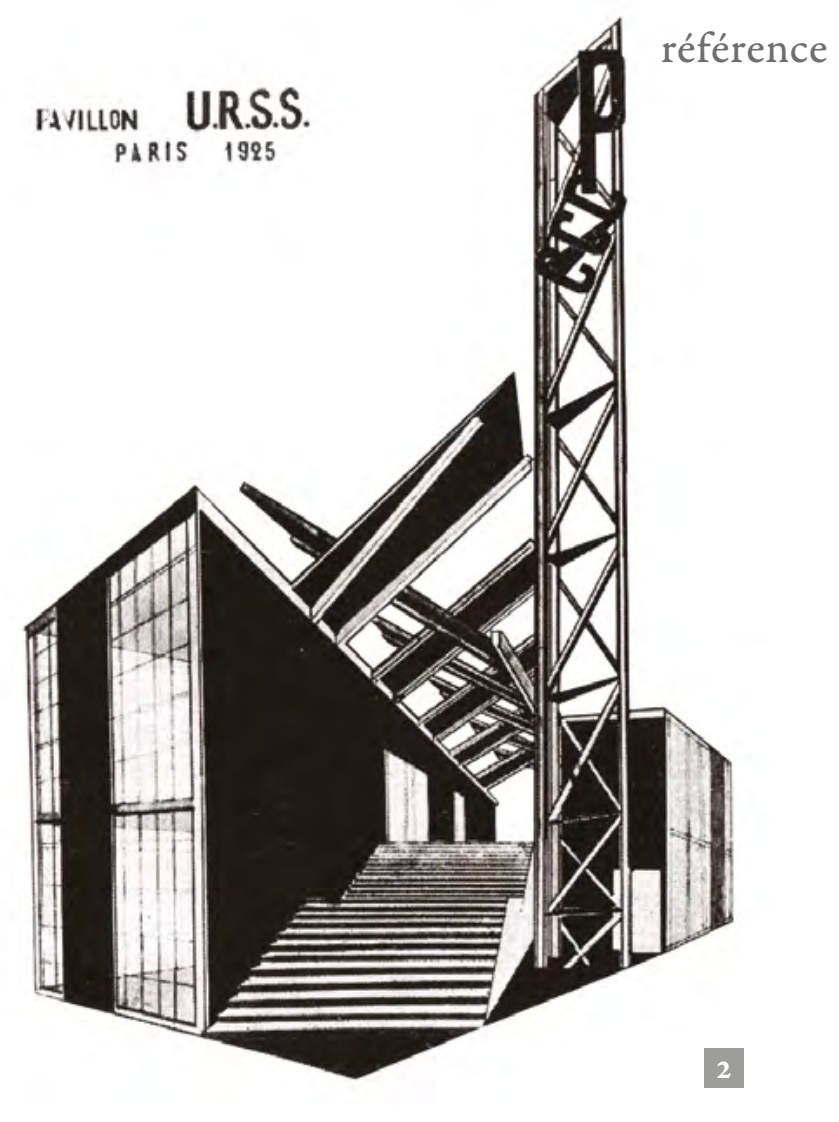

\section{Pavillon URSS [1924-1925]}

Pour les jeunes nations ou régimes qui cherchent à se faire une place sur la carte du monde, au propre comme au figuré, la réalisation de bâtiments représentatifs est la voie royale. La conception d'une telle architecture concorde souvent moins avec les exigences fonctionnelles qu'avec les ambitions de représentation. Le défi consiste généralement à créer une nouvelle monumentalité, capable d'exprimer l'autonomie de la nation.

Dès l'établissement des relations diplomatiques avec la France, fin 1924, I'URSS fut invitée à participer à l'Exposition internationale des arts décoratifs et industriels modernes. ${ }^{2}$ Melnikov conçut et construisit le pavillon qui fut considéré comme l'un des plus novateurs de l'exposition. Délibérément, l'idée était de ne pas s'opposer aux palais luxueux des autres nations, dont l'intérieur dégénérait en galerie commerciale. Le pavillon devait être l'incarnation de l'architecture soviétique, mais aussi de la culture et de la créativité nationale.

En 1924, Melnikov avait acquis une certaine notoriété grâce au projet de sarcophage de Lénine, destiné au mausolée de la Place Rouge ${ }^{3}$.La même année, il remporta le concours qui précédait la commande. II bâtit l'ensemble du terrain de 29,5 sur $11 \mathrm{~m}$. La construction en bois légère impressionna surtout par sa puissance spatiale inhabituelle, malgré un plan clairement ordonné et même une construction symétrique. Le volume rectangulaire est divisé diagonalement par un grand escalier extérieur. Un dispositif de panneaux inclinés protège ce passage public du soleil et de la pluie. Le mât, une structure treillis triangulaire, marque l'accès au pavilIon. À l'intérieur, le plan libre permet de moduler la composition des espaces. La lumière du soleil pénètre en abondance par les généreuses baies vitrées. En tant qu'artiste participant principal, Alexan- der Rodchenko détermina la palette de couleurs.

Les différents composants de l'édifice furent préfabriqués à Moscou, transportés en train jusqu'à Paris, puis assemblés sur place en moins d'un mois par dix ouvriers tout au plus ${ }^{4}$. Cette méthode de travail nécessita une simplification drastique du projet. Après la construction du pavillon, lorsqu'il lui fut demandé de répondre à l'accusation de formalisme pur, Melnikov répondit avec conviction: "Je voulais que le pavillon soit aussi aérien et lumineux que possible : c'est mon choix personnel, mais je pense que cela reflète également la préférence de l'ensemble de notre nation.. Le choix des couleurs, la simplicité des lignes, l'abondance d'air et de lumière, autant de caractéristiques inhabituelles qui peuvent plaire ou non au visiteur, viennent de mon pays. Mais bon sang, n'avez-vous pas encore remarqué que j'ai volontairement bâti un symbole? "5 Le Corbusier, lui-même auteur du PavilIon de l'Esprit Nouveau, estimait que le Pavillon soviétique était le seul qui valait la peine d'être vu à l'exposition. Le Grand Prix fut décerné à Melnikov 6 . L'Union soviétique prouva ainsi qu'au-delà des conceptions utopiques, elle était en mesure de réaliser des projets réels et aptes à être exécutés. Melnikov devint une star dans son pays et à l'étranger.

La pensée moderne et le langage formel qui lui était associé ne cessaient de gagner en importance grâce aux contacts professionnels entre les architectes de toute l'Europe. Une génération montante de constructivistes se réunit à Moscou et fonda la revue Architecture contemporaine (SA), qui diffusait exclusivement les tendances modernes de l'architecture européenne. L'innovation en matière d'architecture devint un facteur de concurrence internationale.
2-Perspective

3-Plan du rez-de-chaussée et de l'étage

2- T. van DOESBURG, On European Architecture, Complete Essays from Het Bouwbedriff 1924-1931, Birkhäuser, 1990, p. 178.

3,4- S. Frederick Starr, K MEL'NIKOv, Le Pavillon Soviétique, L'Equerre, 1981, p. 50,74.

5,6- M. Fosso, O. Macel, M MerigGi, Konstantin S. Mel'nikov and the Construction of Moscow, Skira, 2000, p. 62, 141
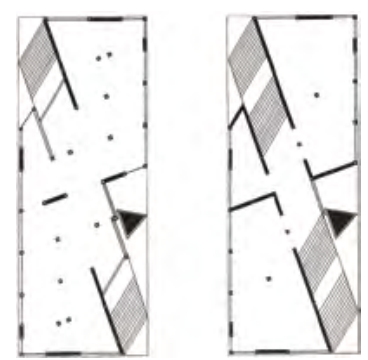

3 


\section{La Maison Melnikov [1927-1929]}

On s'attendait à ce que la conception des immeubles de logement découle directement des changements révolutionnaires, entraînant ensuite un nouveau mode de vie pour chaque individu. En réalité, le collectivisme prometteur portait sur l'adaptation de la population à l'indigence par la mise à disposition d'équipements collectifs. L'offre d'habitations susceptible de répondre aux nouvelles aspirations sociales était affligeante. Dans la construction de logements, la crise généralisée était profonde et les moyens étaient maigres.

Le collectivisme consiste à faire passer l'intérêt de la communauté avant celui de l'individu. II est donc singulier que le patriote Melnikov ait justement construit pour lui-même et sa famille une maison unifamiliale isolée, dans le centre de Moscou. Une typologie qui appuie la hiérarchie public/privé et qui est dans le fond individualiste. L'habitation est généralement considérée comme emblématique de l'architecture soviétique des années vingt. Néanmoins, et c'est paradoxal, l'idéal du socialisme ne peut en aucun cas être une habitation isolée. La maison se désolidarise de son contexte par son emplacement solitaire dans une rue comptant exclusivement des maisons mitoyennes et par sa position en retrait par rapport à l'alignement existant. La silhouette ne peut être qualifiée de traduction archétypique du programme, bien qu'il s'agisse d'une interprétation particulière du concept d'habitation. On pourrait même penser qu'il lui manque une fonction. D'un point de vue volumétrique, le bâtiment est composé de deux cylindres imbriqués l'un dans l'autre. L'intersection forme la charnière qui abrite la circulation. Selon leur position par rapport au niveau du sol et à la rue les espaces reçoivent une fonction différente : les pièces traditionnellement plus vouées à la représentation, à savoir la salle à manger au rez-de-chaussée et le séjour à l'étage sont situées côté rue. Les espaces sanitaires, la chambre à coucher commune et l'atelier se trouvent à l'arrière.

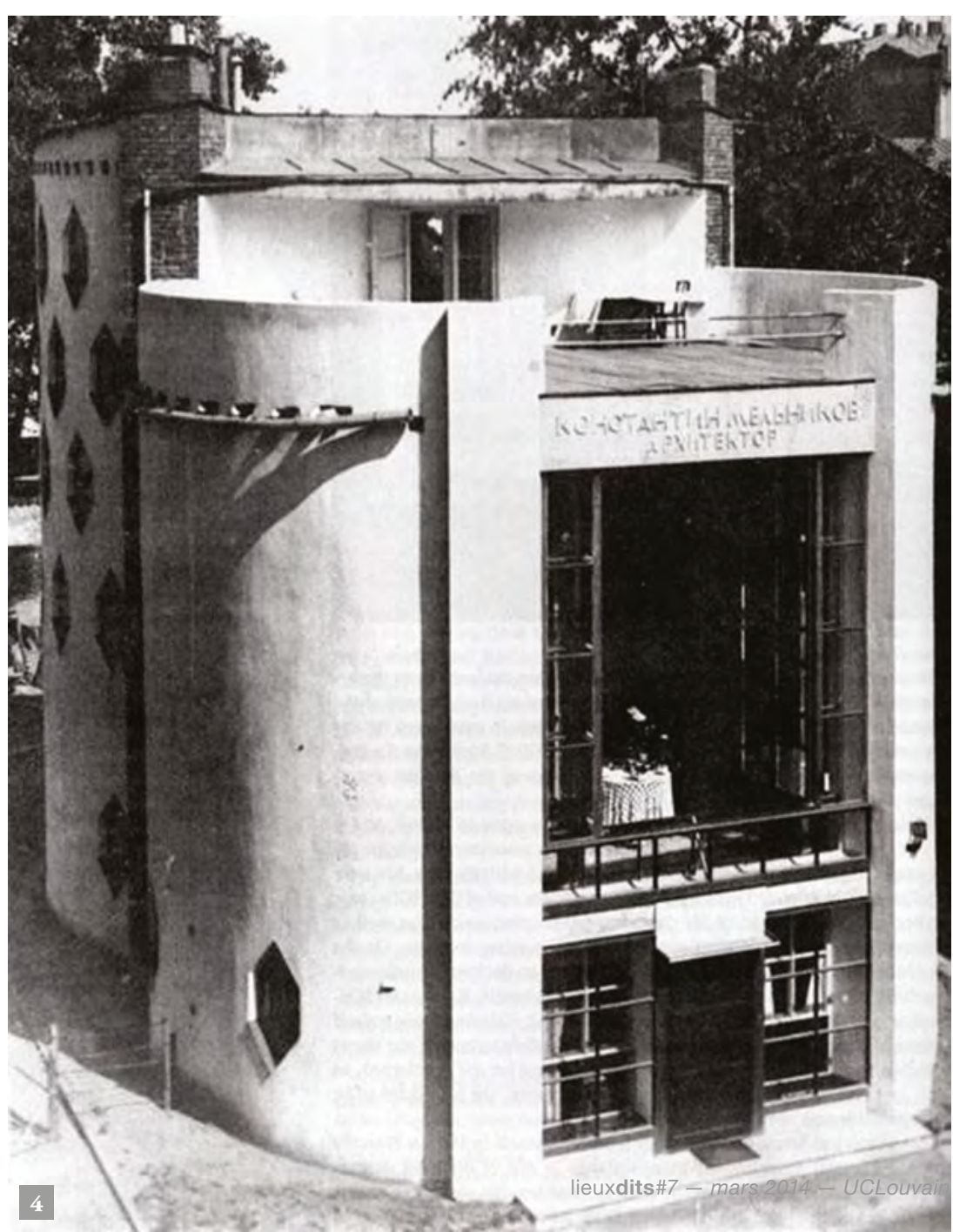


Aucun espace n'est en contact physique direct avec le jardin environnant. Cette répulsion vis-à-vis du terrain naturel est confirmée par la porte d'accès unique côté rue, l'absence totale d'aménagement de l'espace extérieur, le toit en terrasse et la composition de la façade.

Une vaste fenêtre domine la façade avant. Au-dessus trône la signature de l'architecte, en lettres capitales : Konstantin Melnikov, ArCHITECte, qui souligne ainsi l'importance de son nom, de sa personnalité et de sa profession. Malgré la nature collectiviste de la pratique architecturale et de la vie à Moscou à cette époque, il tenait coûte que coûte à conserver son individualité créative.

Disposant de moyens financiers limités, Melnikov se montra particulièrement inventif pour la conception de la structure et l'utilisation des matériaux. La maçonnerie des parois porteuses extérieures, particulièrement originale, présente un maillage diagonal régulièrement percé d'évidements hexagonaux. Cette méthode a rendu superflue l'utilisation de linteaux pour la réalisation des ouvertures. La façade se détache de la logique constructive par le remplissage d'une grande partie du squelette au moyen de déchets de construction. L'enveloppe conventionnelle de l'habitation et ses composants se fondent pour céder la place à la sculpture et à la composition. Petite en soi, la maison a ainsi pu être sublimée au-delà de son contexte.

À l'intérieur aussi, l'utilisation des matériaux n'est pas seulement élémentaire-constructive, mais aussi élémentaire-figurative : la sensation intérieure provient de la lumière sacrée qui pénètre à travers les ouvertures innombrables et relativement petites de la façade.

À la base, Melnikov ne cherchait pas à obtenir une superficie au sol maximale au départ d'un périmètre mural minimal grâce à un plan de pièces circulaire. ${ }^{7}$ II se serait essentiellement concentré sur une méthode originale permettant de fusionner deux volumes simples. Le jeu de lumière confère un aspect sublime à l'intersection qui évoque les expériences transcendantales de Boullée.

Une question demeure : est-il pratique et donc souhaitable de vouloir habiter dans deux cylindres ? L'histoire a effectivement prouvé le contraire. L'habitat fait partie de la réalité quotidienne : ce programme banal n'exige rien d'exceptionnel, et certainement pas d'être élevé au rang de monument. Si Melnikov n'a rien contre l'utilisation et la fonctionnalité, ce bâtiment se révèle toutefois extrêmement exigeant pour ses occupants parce qu'il fait passer l'expérience et le luxe visuel avant le confort et la commodité. L'audace de l'entreprise et le plaisir pur du raffinement formel méritent cependant la plus grande admiration.
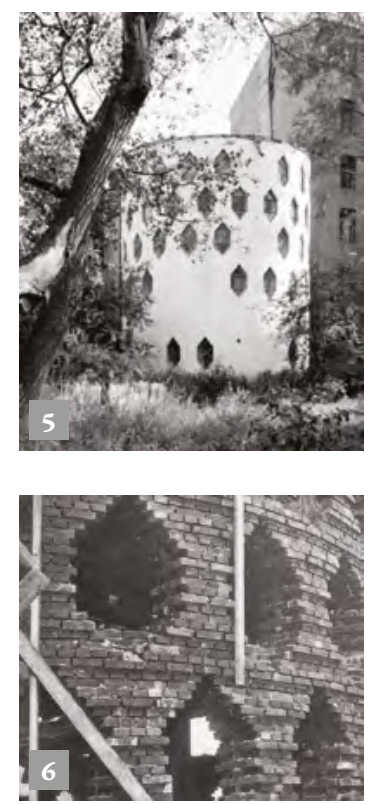

7- KenTadashi Öshima, TOSHIKO KinOSHITA, Visions of the Real, Modern Houses in the 20th Century:1, a+u, 2000, p. 122
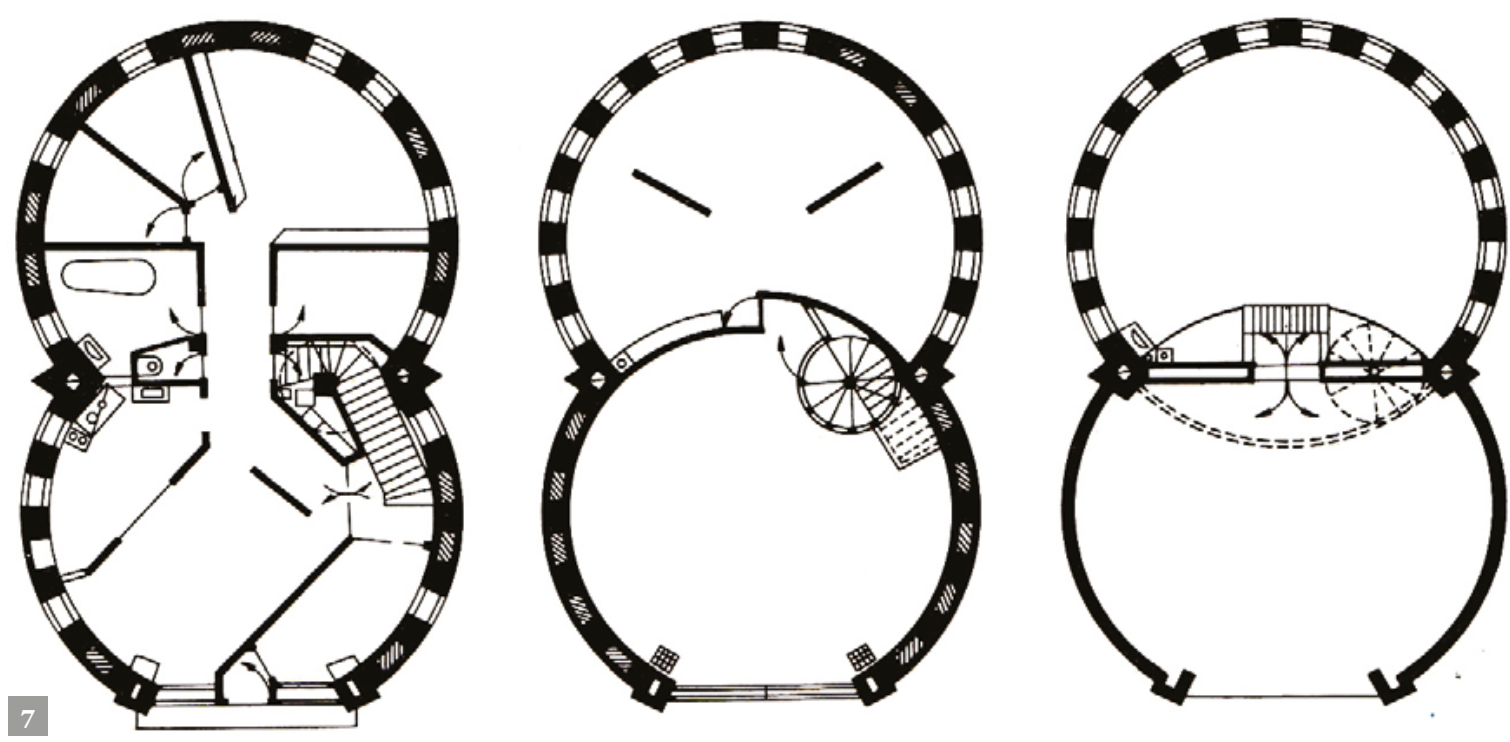


\section{Le Club des travailleurs Roussakov [1927-1929]}

Les Clubs de travailleurs, également connus sous le nom de Maisons du peuple, poussèrent comme des champignons dans les premières années du régime soviétique. Ils devinrent d'importants centres de diffusion de la nouvelle culture socialiste au sein de la classe ouvrière. Simultanément, l'intention était de créer un lien entre les différents moments de la vie quotidienne, en particulier la transition de la dimension collective du travail à l'usine à la dimension individuelle de la vie familiale.

Résultat d'une évolution historique, le paysage de communes isolées autour de Moscou subit la pression de l'extension urbaine $^{8}$. C'est ce délicat tissu semi-urbanisé qui constitua le cadre des expériences de Melnikov : il conçut huit Clubs de travailleurs dans la périphérie industrielle de Moscou, dont six furent effectivement construits. Tous les complexes qu'il imagina renforcèrent la structure d'archipel en imposant un nouvel ordre grâce à des interventions stratégiques, en utilisant aussi bien le contraste et l'assonance que les facteurs de la composition.

Le Club des travailleurs de Roussakov se situe le long d'une route principale, dans un recoin d'un parc. Malgré l'évolution du quartier de Sokol'niki, le bâtiment continue aujourd'hui encore à jouer un rôle important dans le tissu local grâce à son interaction avec l'espace public environnant. Un plan terrier triangulaire issu du théâtre classique est à la base de l'implantation : les sièges sont placés en éventail autour de la scène. Melnikov avait prévu une série de méthodes ingénieuses permettant de convertir le grand hall de 1200 places assises ${ }^{9}$ en petits locaux aptes à accueillir un grand nombre d'activités. Trois tribunes pouvaient être séparées du reste de la salle principale par un dispositif de trappes qui la transformait en auditoriums individuels.

Melnikov a envisagé le club comme un seul volume compact doté d'une forme nettement symétrique, comme à l'ordinaire. L'extérieur expressif doté d'auditoriums en encorbellement est une extériorisation de l'agencement intérieur innovant. Fondamentalement, la forme est une expression de la fonction, avec peu d'espaces perdus, mais elle ne peut être qualifiée de puritaine. Extrêmement simple et ordonné sur le plan, mais tellement ingénieux en termes de construction, le projet reste cohérent du début à la fin. Car si, dans la maison de l'architecte, le mouvement est contenu dans une constellation de corps uniformes, i est ici déchaîné pour donner vie à l'ensemble de la forme dans une composition complexe et très articulée.

Le bâtiment en tant que forme sculpturale donne aujourd'hui une impression beaucoup plus familière qu'au début $d u$ XXe siècle. Le mot formalisme est devenu une insulte. Néanmoins, la forme est propre à l'architecture et on peut parfaitement s'extasier devant une composition simplement formelle. L'architecture de Melnikov est plus profonde qu'on ne pourrait le penser au premier abord. Des raisons d'ordre fonctionnel, symbolique, économique et constructif assurèrent à Melnikov une motivation sous-jacente lors de la création de structures spatiales et volumétriques. Ce qui constitua littéralement sa marque de fabrique !

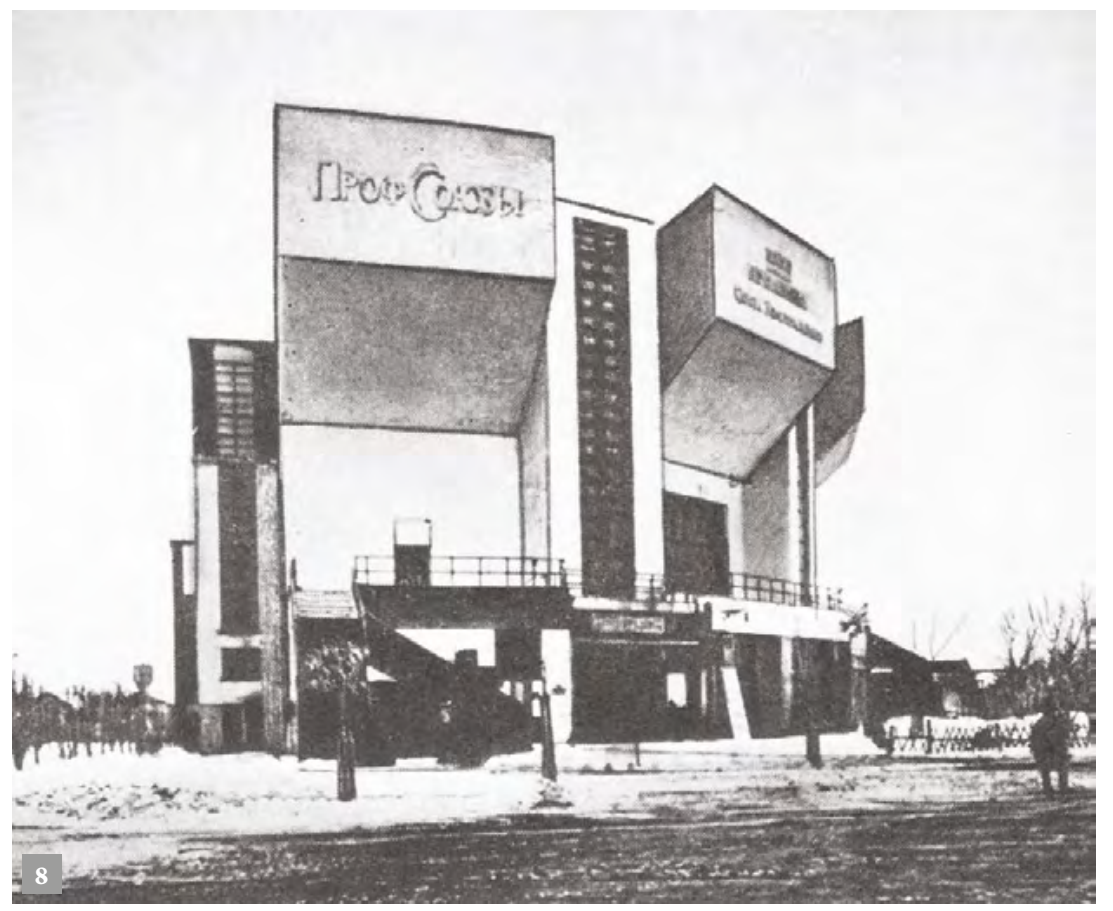




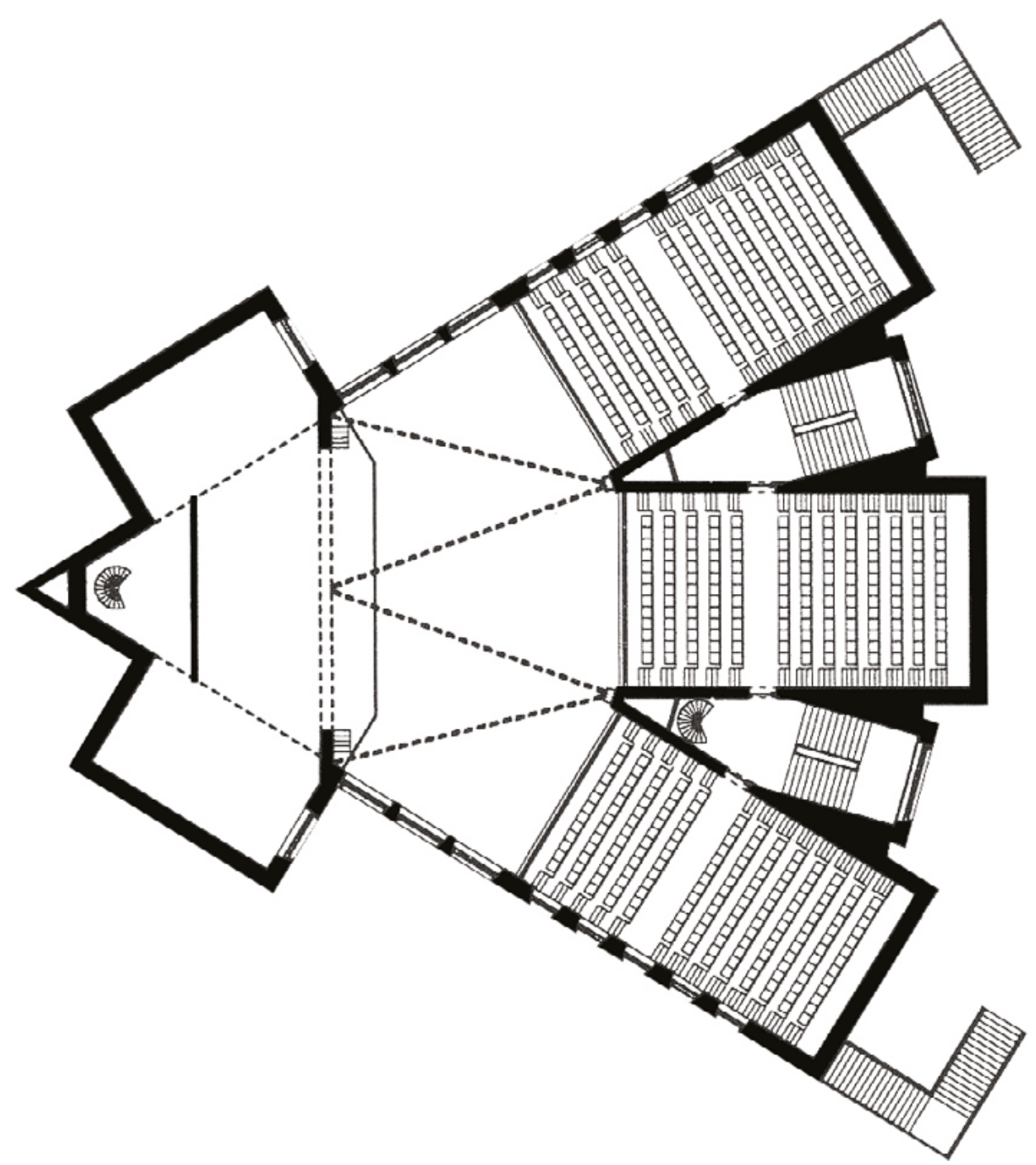

